\title{
Temperature-induced resonances and Landau damping of collective modes in Bose-Einstein condensed gases in spherical traps
}

\author{
M. Guilleumas ${ }^{1}$ and L. P. Pitaevskii, ${ }^{1,2}$ \\ ${ }^{1}$ Dipartimento di Fisica, Università di Trento and Istituto Nazionale per la Fisica della Materia, I-38050 Povo, Italy \\ ${ }^{2}$ Kapitza Institute for Physical Problems, 117454 Moscow, Russian Federation
}

(Received 14 June 1999; published 9 December 1999)

\begin{abstract}
Interaction between collective monopole oscillations of a trapped Bose-Einstein condensate and thermal excitations is investigated by means of perturbation theory. We assume spherical symmetry to calculate the matrix elements by solving the linearized Gross-Pitaevskii equations. We use them to study the resonances of the condensate induced by temperature when an external perturbation of the trapping frequency is applied and to calculate the Landau damping of the oscillations.
\end{abstract}

PACS number(s): 03.75.Fi, 02.70.Lq, 67.40.Db

\section{INTRODUCTION}

Since the discovery of Bose-Einstein condensation in magnetically trapped Bose gases, the study of the low-energy collective excitations has attracted much interest, both from an experimental and a theoretical point of view. Mean-field theory has proven to be a good framework to study static, dynamic, and thermodynamic properties of these trapped gases. In particular, it provides predictions of the frequencies of collective excitations that agree very well with the observed ones. Recently $[1,2]$, the energy shifts and damping rates of these low-lying collective excitations have been measured as a function of temperature. However, these phenomena have not yet been completely understood theoretically.

In this paper we study the influence of thermal excitations on collective oscillations of the condensate in the collisionless regime. Previous papers on this subject have been devoted mainly to calculation of the Landau damping by means of perturbation theory. In Refs. [3-6] only the uniform system has been considered, whereas in Refs. [7,8] Landau damping in trapped Bose gases has also been studied but using the semiclassical approximation for thermal excitations and the hydrodynamic approximation for collective oscillations. An important point of Refs. [7,8] is that the authors discuss the possible chaotic behavior of the excitations in an anisotropic trap. The frequency shift has also been studied for a trapped condensate in the collisionless regime in Ref. [8].

In the present work, we study the interaction between collective and thermal excitations using the Gross-Pitaevskii equation and perturbation theory. We consider spherically symmetric traps, since in this case the spectrum of excitations is easily calculated, avoiding the use of further approximations. Even though the case of anisotropic traps can be significantly different in the final results, a detailed investigation of spherical traps is instructive. We explore, in particular, the properties of monopole oscillations by studying the temperature-induced resonances that occur in the condensate when an external perturbation of the trapping frequency is applied and, also, the Landau damping associated with the interaction with thermally excited states.
This paper is organized as follows. In Sec. II we introduce the general equations that describe the elementary excitations of the condensate within the Bogoliubov theory [9]. In Sec. III we recall the perturbation theory for a trapped Bosecondensed gas in order to study the interaction between elementary excitations. In Sec. IV we introduce the linearresponse function formalism and calculate the response function of the condensate when a small perturbation of the trapping frequency is applied. We derive analytic equations for the response function at zero temperature and treat perturbatively the contribution of the elementary excitations, which is related to Landau damping. In Sec. V we discuss the main results.

\section{ELEMENTARY EXCITATIONS OF AN ISOTROPIC TRAP}

We consider a weakly interacting Bose-condensed gas confined in an external potential $V_{\text {ext }}$ at $T=0$. The elementary excitations of a degenerate Bose gas are associated with the fluctuations of the condensate. At low temperature they are described by the time-dependent Gross-Pitaevskii (GP) equation for the order parameter $[10,11]$ :

$$
i \hbar \frac{\partial}{\partial t} \Psi(\mathbf{r}, t)=\left(-\frac{\hbar^{2} \boldsymbol{\nabla}^{2}}{2 m}+V_{\mathrm{ext}}(\mathbf{r})+g|\Psi(\mathbf{r}, t)|^{2}\right) \Psi(\mathbf{r}, t),
$$

where $\int d \mathbf{r}|\Psi|^{2}=N_{0}$ is the number of atoms in the condensate. At zero temperature it coincides with the total number of atoms $N$, except for a very small difference $\delta N \ll N$ due to the quantum depletion of the condensate. The coupling constant $g$ is proportional to the $s$-wave scattering length $a$ through $g=4 \pi \hbar^{2} a / m$. In the present work we will discuss the case of positive scattering length, as for ${ }^{87} \mathrm{Rb}$ atoms. The trap is included through $V_{\text {ext }}$, which is chosen here in the form of an isotropic harmonic potential: $V_{\text {ext }}(r)$ $=(1 / 2) m \omega_{\mathrm{ho}}^{2} r^{2}$. The harmonic trap provides a typical length scale for the system, $a_{\mathrm{ho}}=\left(\hbar / m \omega_{\mathrm{ho}}\right)^{1 / 2}$.

So far experimental traps have axial symmetry, with different radial and axial frequencies, but experiments with spherical traps are also feasible [12]. The choice here of a spherical trap is due to two different reasons. First, it greatly 
reduces the numerical effort and will allow us to study the interaction of oscillations with elementary excitations without any further approximations. Second, the energy spectrum of the excitations in such a trap is well resolved, yielding to the appearance of well-separated resonances. In anisotropic traps, conversely, the spectrum of excitations is much denser.

The normal modes of the condensate can be found by linearizing Eq. (1), i.e., looking for solutions of the form

$$
\Psi(\mathbf{r}, t)=e^{-i \mu t / \hbar}\left[\Psi_{0}(\mathbf{r})+u(\mathbf{r}) e^{-i \omega t}+\mathrm{V}^{*}(\mathbf{r}) e^{i \omega t}\right],
$$

where $\mu$ is the chemical potential and the functions $u$ and $v$ are the "particle" and "hole" components characterizing the Bogoliubov transformations. After inserting in Eq. (1) and retaining terms up to first order in $u$ and $\mathrm{v}$, one finds three equations. The first one is the nonlinear equation for the order parameter of the ground state,

$$
\left[H_{0}+g \Psi_{0}^{2}(\mathbf{r})\right] \Psi_{0}(\mathbf{r})=\mu \Psi_{0}(\mathbf{r}),
$$

where $H_{0}=-\left(\hbar^{2} / 2 m\right) \nabla^{2}+V_{\text {ext }}(\mathbf{r})$, while $u(\mathbf{r})$ and $\mathbf{v}(\mathbf{r})$ obey the following coupled equations [11]:

$$
\begin{gathered}
\hbar \omega u(\mathbf{r})=\left[H_{0}-\mu+2 g \Psi_{0}^{2}\right] u(\mathbf{r})+g \Psi_{0}^{2} \mathrm{~V}(\mathbf{r}), \\
-\hbar \omega \mathrm{V}(\mathbf{r})=\left[H_{0}-\mu+2 g \Psi_{0}^{2}\right] \mathrm{V}(\mathbf{r})+g \Psi_{0}^{2} u(\mathbf{r}) .
\end{gathered}
$$

Numerical solutions of these equations have been found by different authors [13-18]. In the present work, we use them to calculate the response function of the condensate under an external perturbation and the Landau damping of collective modes.

When the adimensional parameter $N a / a_{\text {ho }}$ is large, the time-dependent GP equation reduces to the hydrodynamic equations [19]:

$$
\begin{gathered}
\frac{\partial \rho}{\partial t}+\boldsymbol{\nabla}(\mathbf{v} \rho)=0 \\
m \frac{\partial}{\partial t} \mathbf{v}+\nabla\left(V_{\mathrm{ext}}+g \rho+\frac{m \mathrm{v}^{2}}{2}\right)=0,
\end{gathered}
$$

where $\rho(\mathbf{r}, t)=|\Psi(\mathbf{r}, t)|^{2}$ is the particle density and the velocity field is $\mathbf{v}(\mathbf{r}, t)=\left(\Psi^{*} \nabla \Psi-\Psi \nabla \Psi^{*}\right) \hbar /(2 m i \rho)$. The static solution of Eqs. (6) and (7) gives the Thomas-Fermi ground-state density, which in the spherical symmetric trap reads

$$
\rho(r)=g^{-1}\left[\mu-V_{\mathrm{ext}}(r)\right]
$$

in the region where $\mu>V_{\text {ext }}(r)$, and $\rho=0$ elsewhere. The chemical potential $\mu$ is fixed by the normalization of the density to the number of particles $N_{0}$ in the condensate. The density profile (8) has the form of an inverted parabola, which vanishes at the classical turning point $R$ defined by the condition $\mu=V_{\text {ext }}(R)$. For a spherical trap, this implies

$$
\mu=\frac{m \omega_{\mathrm{ho}}^{2} R^{2}}{2} .
$$

It has been shown [19] that the hydrodynamic equations (6) and (7) correctly reproduce the low-lying normal modes of the trapped gas in the linear regime when $N a / a_{\text {ho }}$ is large (see, however, Ref. [20]).

\section{PERTURBATION THEORY}

Let us briefly recall the perturbation theory for the interaction between collective modes of a condensate and thermal excitations as it was developed in Ref. [4]. Suppose that a certain mode of the condensate has been excited and, therefore, it oscillates with the corresponding frequency $\Omega_{\text {osc }}$. We assume that this oscillation is classical, i.e., the number of quanta of oscillation $\left(n_{\text {osc }}\right)$ is very large. Then, the energy of the system associated with the occurrence of this classical oscillation can be calculated as $E=\hbar \Omega_{\text {osc }} n_{\text {osc }}$ with $n_{\text {osc }} \gg 1$. Due to interaction effects, the thermal bath can either absorb or emit quanta of this mode producing a damping of the collective oscillation. The energy loss can be written as

$$
\dot{E}=-\hbar \Omega_{\text {osc }}\left(W^{(a)}-W^{(e)}\right),
$$

where $W^{(a)}$ and $W^{(e)}$ are the probabilities of absorption and emission of one quantum $\hbar \Omega_{\mathrm{osc}}$, respectively. The interaction between excitations is small, so one can use perturbation theory to calculate the probabilities for the transition between an $i$ th excitation and a $k$ th one, available by thermal activation

$$
W=\pi \sum_{i, k}\left|\left\langle k\left|V_{\text {int }}\right| i\right\rangle\right|^{2} .
$$

Let $E_{i}$ and $E_{k}$ be the corresponding energies and assume $E_{k}>E_{i}$. Since energy is conserved during the transition process, one has $E_{k}=E_{i}+\hbar \Omega_{\text {osc }}$.

The interaction term in second quantization is given by

$$
V_{\text {int }}=\frac{g}{2} \int d \mathbf{r} \hat{\Psi}^{\dagger} \hat{\Psi}^{\dagger} \hat{\Psi} \hat{\Psi} .
$$

In the framework of Bogoliubov theory, the field operator $\hat{\Psi}$ can be written as the sum of the condensate wave function $\Psi_{0}$, which is the order parameter at equilibrium, and its fluctuations $\delta \hat{\Psi}$, where $\hat{\Psi}=\Psi_{0}+\delta \hat{\Psi}$ [see Eq. (2)]. The fluctuations can be expressed in terms of the annihilation $(\alpha)$ and creation $\left(\alpha^{\dagger}\right)$ operators of the elementary excitations of the system:

$$
\delta \hat{\Psi}=\sum_{j}\left[u_{j}(\mathbf{r}) \alpha_{j}+\mathrm{v}_{j}^{*}(\mathbf{r}) \alpha_{j}^{\dagger}\right],
$$

where the functions $u$ and $\mathrm{V}$ are properly normalized solutions of Eqs. (4) and (5). In the sum (13), one can select a low-energy collective mode, for which we use the notation $u_{\mathrm{osc}}, \mathrm{v}_{\mathrm{osc}}, \alpha_{\mathrm{osc}}, \alpha_{\mathrm{osc}}^{\dagger}$, and investigate its interaction with higher-energy single-particle excitations, for which we use the indices $i, k$ as in Eq. (11). These latter excitations are assumed to be thermally excited. Inserting expression (13) into Eq. (12), one rewrites the interaction term $V_{\text {int }}$ in terms 
of the annihilation and creation operators. Since we want to study the decay process in which a quantum of oscillation $\hbar \Omega_{\mathrm{osc}}$ is annihilated (created) and the $i$ th excitation is transformed into the $k$ th one (or vice versa), we will keep only terms linear in $\alpha_{\text {osc }}\left(\alpha_{\text {osc }}^{\dagger}\right)$ and in the product $\alpha_{k}^{\dagger} \alpha_{i}\left(\alpha_{k} \alpha_{i}^{\dagger}\right)$. And the energy conservation during the transition process will be ensured by the delta function $\delta\left(E_{k}-E_{i}-\hbar \Omega_{\text {osc }}\right)$. This mechanism is known as Landau damping [21].

Assuming that at equilibrium the states $i, k$ are thermally occupied with the usual Bose factor $f_{i}=\left[\exp \left(E_{i} / k_{B} T\right)\right.$ $-1]^{-1}$, the rate of energy loss can be calculated as [4]

$$
\dot{E}=-2 \pi \frac{E}{\hbar} \sum_{i k}\left|A_{i k}\right|^{2} \delta\left(E_{k}-E_{i}-\hbar \Omega_{\mathrm{osc}}\right)\left(f_{i}-f_{k}\right),
$$

where

$$
\begin{aligned}
A_{i k}= & 2 g \int d \mathbf{r} \psi_{0}\left[\left(u_{k}^{*} \mathrm{v}_{i}+\mathrm{v}_{k}^{*} \mathrm{v}_{i}+u_{k}^{*} u_{i}\right) u_{\mathrm{osc}}\right. \\
& \left.+\left(\mathrm{v}_{k}^{*} u_{i}+\mathrm{v}_{k}^{*} \mathrm{v}_{i}+u_{k}^{*} u_{i}\right) \mathrm{v}_{\mathrm{osc}}\right] .
\end{aligned}
$$

Let us define the dissipation rate $\gamma$ through the following relation between the energy of the system $E$ and its dissipation $\dot{E}$ :

$$
\dot{E}=-2 \gamma E \text {. }
$$

Using expression (14), $\gamma$ can be calculated as

$$
\frac{\gamma}{\Omega_{\mathrm{osc}}}=\sum_{i k} \gamma_{i k} \delta\left(\omega_{i k}-\Omega_{\mathrm{osc}}\right),
$$

where the transition frequencies $\omega_{i k}=\left(E_{k}-E_{i}\right) / \hbar$ are positive. The "damping strength"

$$
\gamma_{i k}=\frac{\pi}{\hbar^{2} \Omega_{\mathrm{osc}}}\left|A_{i k}\right|^{2}\left(f_{i}-f_{k}\right)
$$

has the dimensions of a frequency. In this work we calculate the quantities $\gamma_{i k}$ by using the numerical solutions $u$ and $v$ of Eqs. (2)-(5) in the integrals (15). The results will be discussed in Sec. V.

\section{RESPONSE FUNCTION}

The results of the preceding section can be used also to study the effect that an external perturbation of the trap has on the collective excitations of the condensate. Let us assume the trapping frequency in the form $\left[\omega_{\mathrm{ho}}+\delta \omega_{\mathrm{ho}}(t)\right]$, where $\delta \omega_{\mathrm{ho}} \sim \exp (-i \omega t)$ is a time-dependent modulation. Assuming that the perturbation is small, one can use the response function formalism to describe the fluctuations of the system. Let us briefly recall the basic formalism [22].

The behavior of a system under an external perturbation can be described by studying the fluctuations that may generate the external interaction to a certain physical quantity of the system. An external perturbation acting on the system is described by a new term in the Hamiltonian of the type

$$
\hat{V}=-\hat{x} f(t),
$$

where $\hat{x}$ is the quantum operator of the physical quantity that may fluctuate, and $f(t)$ is the "perturbing force." The mean value $\langle x\rangle$ is zero in the equilibrium state, in the absence of perturbation, and is not zero when it is present. For a periodic perturbation $f(t) \sim \exp (-i \omega t)$, the relation between $\langle x\rangle$ and $f(\omega)$ is

$$
\langle x\rangle=\alpha(\omega) f,
$$

where $\alpha(\omega)$ is the response function also called generalized susceptibility.

In general, $\alpha(\omega)$ is a complex function. It can be seen that the imaginary part of the susceptibility determines the absorption of energy $Q$ of the external force $f$ by the system through the relation

$$
Q=\frac{\omega}{2} \operatorname{Im}[\alpha(\omega)]|f|^{2},
$$

and that the real and imaginary parts of $\alpha(\omega)$ satisfy the Kramers-Krönig relation

$$
\operatorname{Re}[\alpha(\omega)]=\frac{2}{\pi} P \int_{0}^{\infty} \frac{\operatorname{Im}[\alpha(\xi)]}{\xi^{2}-\omega^{2}} \xi d \xi
$$

where $P$ means the principal value of the integral.

The time-dependent external drive $\delta \omega_{\text {ho }}$ induces oscillations of the condensate density $\delta \rho$ with frequency $\omega$; $\rho(r, t)=\rho(r, 0)+\delta \rho$. Expanding the energy due to the confining potential, $E_{\mathrm{ho}}=\int V_{\text {ext }} \rho d \mathbf{r}$, with respect to $\delta \omega_{\text {ho }}$ and $\delta \rho$ one obtains the "mixed" term, corresponding to the Hamiltonian (19):

$$
V=m \omega_{\mathrm{ho}} \delta \omega_{\mathrm{ho}} \int r^{2} \delta \rho d \mathbf{r} .
$$

Comparing it with Eq. (19), one can identify the perturbing force and the corresponding coordinate as

$$
f=-m \omega_{\text {ho }} \delta \omega_{\text {ho }}, \quad x=\int r^{2} \delta \rho(r, t) d \mathbf{r} .
$$

Note that the first-order term $m \omega_{\text {ho }} \delta \omega_{\text {ho }} \int r^{2} \rho(r, 0) d \mathbf{r}$ can be omitted because it gives an additive shift in the Hamiltonian which does not contribute to the equations of motion of the system.

Once we have identified $f$ and $x$, we can calculate the response function of the condensate $\alpha(\omega)$. According to the definition one has

$$
x=\alpha(\omega) f .
$$

Let us present the response function in the form $\alpha(\omega)$ $=\alpha_{0}(\omega)+\alpha_{1}(\omega)$, where $\alpha_{0}(\omega)$ corresponds to the response function of the condensate at $T=0$, i.e., calculated without elementary excitations, and $\alpha_{1}(\omega)$ is the contribution of the excitations. At low temperatures it can be assumed that $\alpha_{1}(\omega) \ll \alpha_{0}(\omega)$, and then $\alpha_{1}$ can be treated as a perturbation. 
We proceed as follows. First, we use the hydrodynamic approximation to obtain the response function at $T=0$. Then, within a perturbation theory, we introduce the contribution of the elementary excitations at finite $T$ to obtain $\alpha_{1}(\omega)$.

\section{A. Calculation of $\alpha_{0}(\omega)$ at $T=0$}

For a spherically symmetric breathing mode [23], one can easily prove that the hydrodynamic equations of motion (6) and (7) admit analytic solutions of the form [24]

$$
\rho(r, t)=a_{0}(t)-a_{r}(t) r^{2}, \quad \mathrm{v}(r, t)=\alpha_{r}(t) r .
$$

These equations are restricted to the region where $\rho \geqslant 0$. Notice that they include the ground-state solution (8) in the Thomas-Fermi limit. This is recovered by setting $\alpha_{r}=0, a_{r}$ $=m \omega_{\mathrm{ho}}^{2} /(2 g)$, and $a_{0}=\mu / g$. Inserting Eqs. (26) into the hydrodynamic equations, one obtains two coupled differential equations for the time-dependent coefficients $a_{r}(t)$ and $\alpha_{r}(t)$, while at any time $a_{0}=-(15 N / 8 \pi)^{2 / 5} a_{r}^{3 / 5}$ is fixed by the normalization of the density to the total number of atoms. The form (26) for the density and velocity distributions is equivalent to a scaling transformation of the order parameter. That is, at each time, the parabolic shape of the density is preserved, while the classical radius $R$, where the density (26) vanishes, scales in time as [25]

$$
R(t)=R(0) b(t)=\sqrt{\frac{2 \mu}{m \omega_{\mathrm{ho}}^{2}}} b(t),
$$

where the unperturbed radius $R(0)$ is given by Eq. (9).

The relation between the scaling parameter $b(t)$ and the coefficient $a_{r}(t)$ is $a_{r}=m \omega_{\mathrm{ho}}^{2} /\left(2 g b^{5}\right)$. Inserting it into Eq. (26), we obtain

$$
\rho(r, t)=-\frac{1}{g}\left[m \omega_{\mathrm{ho}}^{2} r^{2} \frac{1}{2 b^{5}}-\mu \frac{1}{b^{3}}\right] .
$$

The hydrodynamic equations then yield $\alpha_{r}=\dot{b} / b$ and

$$
\ddot{b}+\left[\omega_{\mathrm{ho}}+\delta \omega_{\mathrm{ho}}(t)\right]^{2} b-\frac{\omega_{\mathrm{ho}}^{2}}{b^{4}}=0 .
$$

The second and third terms of Eq. (29) give the effect of the external trap and of the interatomic forces, respectively. From Eqs. (27) and (29) it follows that at equilibrium $b=1$ and $\dot{b}=0$. For a small driving strength $\delta \omega_{\text {ho }}$, one can assume that the radius of the cloud is perturbed around its equilibrium value, so

$$
R(t)=R(0)+\delta R(t), \quad b(t)=1+\delta b(t),
$$

where

$$
\delta b(t)=\frac{\delta R(t)}{R(0)} .
$$

This means that $\delta b$ is the fractional amplitude of oscillations of the radius and, therefore, it is a measurable quantity.

In the small-amplitude limit, one can linearize Eq. (29) with respect to $\delta \omega_{\text {ho }}$ and $\delta b$ yielding the following equation:

$$
\delta \ddot{b}+5 \omega_{\text {ho }}^{2} \delta b=-2 \omega_{\text {ho }} \delta \omega_{\text {ho }} .
$$

The solution is

$$
\delta b(t)=\frac{-2 \omega_{\mathrm{ho}}}{\Omega_{M}^{2}-\omega^{2}} \delta \omega_{\mathrm{ho}},
$$

where $\Omega_{M}=\sqrt{5} \omega_{\text {ho }}$ corresponds to the frequency of the normal mode of monopole in the hydrodynamic limit [19].

Keeping only the lowest order in the small perturbation $\delta b$, Eq. (28) yields

$$
\rho(r, t)=\rho(r, 0)+\frac{1}{g}\left[5 \frac{1}{2} m \omega_{\mathrm{ho}}^{2} r^{2}-3 \mu\right] \delta b,
$$

and using the Thomas-Fermi radius at equilibrium (9), it follows that the density fluctuation is given by

$$
\begin{aligned}
\delta \rho(r, t) & =\rho(r, t)-\rho(r, 0) \\
& =\frac{5 \mu}{g}\left[\left(\frac{r}{R(0)}\right)^{2}-\frac{3}{5}\right] \delta b(t) .
\end{aligned}
$$

We can calculate now $x$ using Eqs. (24) and (35), finding

$$
x=C \delta b(t),
$$

where $C=16 \pi \mu R(0)^{5} /(35 g)$. Then, from Eq. (33), one gets

$$
x=\frac{-2 C \omega_{\mathrm{ho}}}{\Omega_{M}^{2}-\omega^{2}} \delta \omega_{\mathrm{ho}} .
$$

At $T=0$ there are no thermally excited states and, hence, $\alpha(\omega)=\alpha_{0}(\omega)$. By comparing the definition (25) with Eq. (37), one has

$$
\alpha_{0}(\omega)=\frac{2 C}{m\left(\Omega_{M}^{2}-\omega^{2}\right)} .
$$

This is the response function at zero temperature without including any dissipation. Therefore, $\alpha_{0}(\omega)$ is real, i.e., the induced oscillations at $T=0$ are undamped.

The energy of oscillation can be calculated as twice the mean kinetic energy associated to the mode, $E$ $=\int d \mathbf{r} \rho(r, 0) \mathrm{V}^{2}$. For a monopole mode in an isotropic trap, the calculation [23,24] gives $E=\frac{15}{7} N \mu|\delta b|^{2}$, where $|\delta b|$ is the amplitude of the oscillation of the cloud (31). Using Eqs. (36) and (25) at $T=0$, it follows that

$$
E=\frac{15}{7 C^{2}} \mu N\left|\alpha_{0}(\omega) f\right|^{2}
$$




\section{B. Calculation of $\alpha_{1}(\omega)$}

Now we want to calculate the contribution of the thermally excited states to the response function. We study the low-temperature regime, where $\alpha_{1} \ll \alpha_{0}$ and the energy of oscillation (39) can be estimated using $\alpha_{0}$ instead of $\alpha(\omega)$ $=\alpha_{0}(\omega)+\alpha_{1}(\omega)$. The effect of $\alpha_{1}$ will be introduced within a perturbation theory.

We have already seen that the thermal excitations can either absorb or emit quanta of oscillation $\hbar \omega$ and thus they will dissipate energy. The contribution of the elementary excitations to the susceptibility will be a complex function, $\alpha_{1}(\omega)=\operatorname{Re}\left[\alpha_{1}\right]+i \operatorname{Im}\left[\alpha_{1}\right]$, whose imaginary part is related to the absorption of energy $Q$ of the external perturbation. However, in a stationary solution which is the case under consideration, the absorption $Q$ must be compensated by the energy dissipation (16) due to the interaction with the elementary excitations. Therefore,

$$
Q+\dot{E}=0 \text {. }
$$

Let us rewrite the definition of the damping rate (16) by using Eqs. (17) and (18) with a generic oscillation frequency $\omega$ :

$$
\dot{E}=-2 \omega \sum_{i k} \gamma_{i k} \delta\left(\omega_{i k}-\omega\right) E
$$

Inserting Eq. (39) and defining $\beta(\omega)=\alpha_{0}(\omega) / C$ $=2 /\left[m\left(\Omega_{M}^{2}-\omega^{2}\right)\right]$, one obtains the energy dissipation

$$
\dot{E}=-2 \omega \frac{15 \mu N}{7} \sum_{i k} \gamma_{i k} \delta\left(\omega_{i k}-\omega\right)|\beta(\omega)|^{2}|f|^{2} \text {. }
$$

Let us recall that the energy dissipation according to Eqs. (21) and (40) can be calculated also from the imaginary part of the response function $\alpha(\omega)=\alpha_{0}(\omega)+\alpha_{1}(\omega)$. Since $\alpha_{0}(\omega)$ is real, Eq. (21) becomes

$$
Q=\frac{\omega}{2} \operatorname{Im}\left[\alpha_{1}(\omega)\right]|f|^{2}=-\dot{E}
$$

Comparing Eqs. (42) and (43), one can calculate the imaginary part of $\alpha_{1}(\omega)$ as

$$
\operatorname{Im}\left[\alpha_{1}(\omega)\right]=4 \frac{15 \mu N}{7} \sum_{i k} \gamma_{i k} \delta\left(\omega_{i k}-\omega\right)|\beta(\omega)|^{2},
$$

and using the Kramers-Krönig relation (22) one finds the real part

$$
\operatorname{Re}\left[\alpha_{1}(\omega)\right]=\frac{8}{\pi} \sum_{i k} \frac{\omega_{i k} \gamma_{i k}}{\omega_{i k}^{2}-\omega^{2}} \frac{15 \mu N}{7}\left|\beta\left(\omega_{i k}\right)\right|^{2}
$$

Now we have all the ingredients to calculate the response function of a spherically symmetric trapped condensate when the monopole mode is excited and a small perturbation of the trapping frequency $\delta \omega_{\text {ho }} \sim \exp (-i \omega t)$ is applied. It can be calculated within first-order perturbation as $\alpha(\omega)=\alpha_{0}(\omega)$ $+\operatorname{Re}\left[\alpha_{1}(\omega)\right]+i \operatorname{Im}\left[\alpha_{1}(\omega)\right]$, by using Eqs. (38), (45), and
(44), respectively. It is worth stressing that the real part of the susceptibility diverges at $\Omega_{M}$ (resonance of the condensate at $T=0$ ) but also at $\omega_{i k}$, which are the frequencies of the thermal excited modes that due to the interaction are coupled with the monopole.

Actually, the resonances of the condensate can be found by measuring the fractional amplitude of oscillations of the cloud radius $\delta b$ at different perturbing frequencies. This measurable quantity can be easily related to the response function $\alpha(\omega)$ from Eqs. (36) and (25),

$$
\delta b=-\alpha(\omega) \frac{m \omega_{\mathrm{ho}}}{C} \delta \omega_{\mathrm{ho}} .
$$

Note that the perturbation theory we have used is valid when $\left|\alpha_{1}\right| \ll\left|\alpha_{0}\right|$. This condition becomes very restrictive at $\omega$ near $\Omega_{M}$. However, it is not difficult to improve the approximation in this region by taking the benefit of the analogy between the response function and the Green function $G$.

It is well known that the Green function obeys the Dyson equation [26], which relates the perturbed quantity $(G)$ and the unperturbed one $\left(G_{0}\right)$ through the inverse functions $\left(G^{-1}\right.$ and $G_{0}^{-1}$ ) in such a way that a perturbation theory for $G^{-1}$ has a wider applicability than for $G$. Analogously, we will find a relation between the inverse response functions, perturbed $\left(\alpha^{-1}\right)$ and unperturbed $\left(\alpha_{0}^{-1}\right)$. One has

$$
\frac{1}{\alpha}=\frac{1}{\left(\alpha_{0}+\alpha_{1}\right)}=\frac{1}{\alpha_{0}\left(1+\alpha_{1} / \alpha_{0}\right)}
$$

and formally with the same accuracy

$$
\begin{aligned}
\frac{1}{\alpha} & \simeq \frac{1}{\alpha_{0}}\left(1-\frac{\alpha_{1}}{\alpha_{0}}\right) \\
& =\frac{m}{2 C}\left(\Omega_{M}^{2}-\omega^{2}\right)-\frac{8}{\pi} \sum_{i k} \frac{\omega_{i k} \gamma_{i k}}{\omega_{i k}^{2}-\omega^{2}} \frac{15 \mu N}{7 C^{2}} .
\end{aligned}
$$

Now the applicability of Eq. (48) is restricted only by the condition that the second term is small compared to $(m / 2 C) \Omega_{M}^{2}$.

It is worth noting that according to Eq. (48) the poles of $\alpha(\omega)$ related to the resonances are shifted compared to frequencies $\omega_{i k}$ and are given by the equation $\alpha_{1}\left(\omega_{R}^{\prime}\right) / \alpha_{0}\left(\omega_{R}^{\prime}\right)=1$. However, these shifts are very small.

\section{RESULTS}

In order to present numerical results, we choose a gas of ${ }^{87} \mathrm{Rb}$ atoms (scattering length $a=5.82 \times 10^{-7} \mathrm{~cm}$ ). For the spherical trap we fix the frequency $\omega_{\text {ho }}=2 \pi 187 \mathrm{~Hz}$, which is the geometric average of the axial and radial frequencies of Ref. [1] and corresponds to the oscillator length $a_{\text {ho }}=0.791 \times 10^{-4} \mathrm{~cm}$. We solve the linearized GrossPitaevskii equations (2)-(5) at zero temperature to obtain the ground-state wave function $\Psi_{0}$ and the spectrum of excited states $E_{i}$ as well as the corresponding functions $u_{i}(\mathbf{r}), \mathrm{v}_{i}(\mathbf{r})$. In spherically symmetric traps, the eigenfunctions are labeled by $i=(n, l, m)$, where $n$ is the number of nodes in the 
radial solution, $l$ is the orbital angular momentum, and $m$ its projection. The eigenfunctions are $u_{n l m}(\mathbf{r})$ $=U_{n l}(r) Y_{l m}(\theta, \varphi)$, the energies $E_{n l}$ are $(2 l+1)$ degenerate, and the occupation of the thermally excited states is fixed by the Bose factor.

For a fixed number of trapped atoms, $N$, the number of atoms in the condensate, $N_{0}$, depends on temperature $T$. At zero temperature all the atoms are in the condensate, except a negligible quantum depletion [18]. At finite temperature the condensate atoms coexist with the thermal bath. In the thermodynamic limit [27] the $T$ dependence of the condensate fraction is $N_{0}(T)=N\left[1-\left(T / T_{c}^{0}\right)^{3}\right]$.

We consider the collective excitations in the collisionless regime. This regime is achieved at low enough temperature. The excitation spectrum at low temperature can be safely calculated by neglecting the coupling between the condensate and thermal atoms [28]. It means that the excitation energies at a given $T$ can be obtained within Bogoliubov theory at $T=0$ normalizing the number of condensate atoms to $N_{0}(T)$.

We investigate the monopole mode $(l=m=0$ and $n$ $=1$ ). The functions $u_{\mathrm{osc}}$ and $\mathrm{v}_{\mathrm{osc}}$ do not present angular dependence, and from Eq. (15) it is straightforward to see that the matrix element $A_{i k}$ couples only those energy levels $(i, k)$ with the same quantum numbers $l$ and $m$. That is, the selection rules corresponding to the monopolelike transition are $\Delta l=0$ and $\Delta m=0$. It is obvious, also, that different pairs of levels with the same quantum numbers $n$ and $l$ but different $m$ give the same contribution. Therefore, only the integration of the radial part has to be done numerically.

With fixed $N_{0}$ and at a given temperature, we calculate the damping strengths (18) for the transitions $\omega_{i k}$ coupled with the monopole. In Fig. 1, we show the values of $\gamma_{i k}$ (in units of $\omega_{\text {ho }}$ ) for $N_{0}=50000{ }^{87} \mathrm{Rb}$ atoms at $k_{B} T=\mu$. The arrow points to the frequency of the breathing mode $\Omega_{M}$ $=2.231 \omega_{\text {ho }}$, and the chemical potential is $\mu=15.69 \hbar \omega_{\text {ho }}$ [these values are numerical results of the linearized GrossPitaevskii equations (2)-(5) for $N_{0}=50000$ rubidium atoms]. The position of the bars corresponds to the allowed transition frequencies $\omega_{i k}$ (in units of $\omega_{\mathrm{ho}}$ ), whereas their height defines the numerical value of $\gamma_{i k}$ [29].

One can see that there are two different types of allowed transitions $\omega_{i k}$. The damping strength associated to most of them is very small. Conversely, there are a few transitions which give relatively large values of $\gamma_{i k}$. The latter correspond to transitions between the lowest levels $\left(n_{k}=1, n_{i}\right.$ $=0)$ for different values of $l(l=2,3,4,5)$. The main reason for these "strong transitions" is that the temperature occupation factor for these low-lying levels is large. Moreover, the calculation shows that the matrix elements are also enhanced compared to other transitions. This is due to the fact that the radial wave functions involved in the integration have either one $\left(n_{k}=1\right)$ or no node $\left(n_{i}=0\right)$, contrary to the oscillating character of the radial wave functions associated to higher levels [20].

The contribution of the other transitions is like a small "background" which is difficult to resolve in the scale of the figure. A close-up view of the damping strengths of the tran-

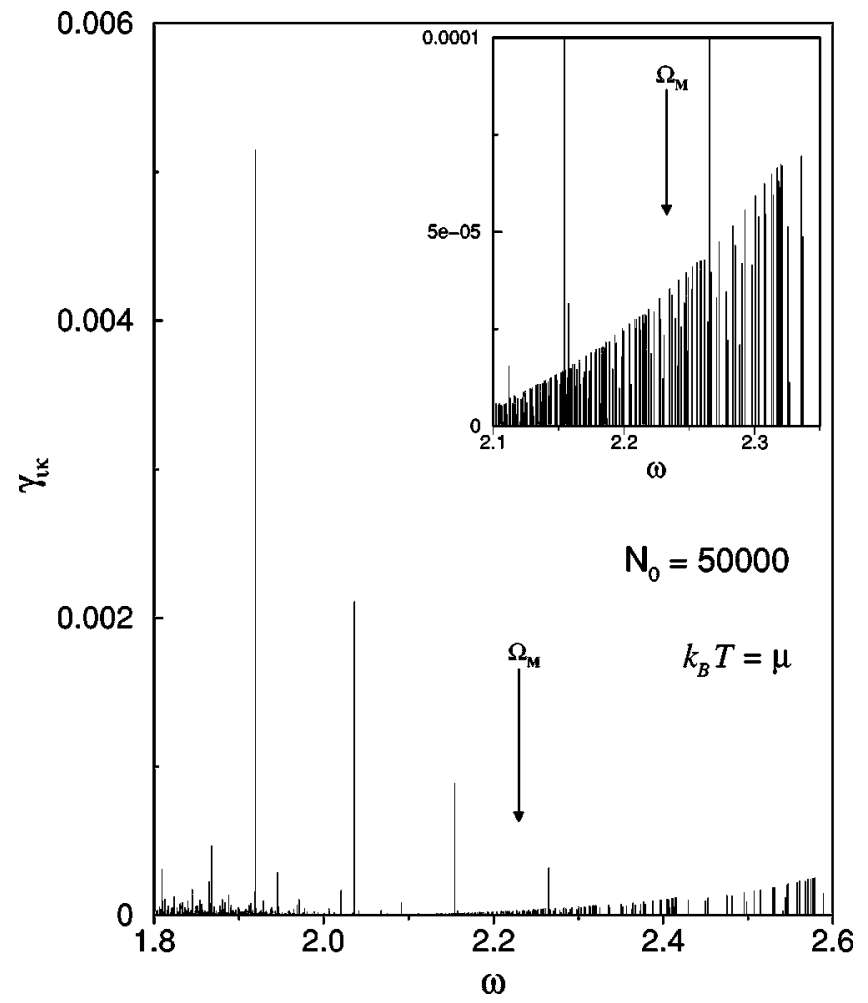

FIG. 1. Transition frequencies $\omega_{i k}$ (in units of $\omega_{\text {ho }}$ ) allowed by the monopole selection rules, for $N_{0}=50000$ atoms of ${ }^{87} \mathrm{Rb}$ in a spherical trap with $a_{\mathrm{ho}}=0.791 \times 10^{-4} \mathrm{~cm}$, at $k_{B} T=\mu$. The vertical bars have length equal to the corresponding damping strength (in units of $\omega_{\text {ho }}$ ). The arrow points to the monopole frequency $\Omega_{M}$. A close-up view of the transition frequencies around $\Omega_{M}$ is presented in the inset in order to show the dense background.

sition frequencies around the monopole is displayed in the inset of Fig. 1 in order to show the dense background. It is worth stressing that such a distinction between "background" and "strong" transitions depends on the number of condensed atoms in the system and, of course, on temperature. When the number of atoms in the condensate increases, the number of excited states available by thermal excitations also increases, leading to a denser and less resoluble background.

In Fig. 2 we present the same as in Fig. 1 but for $N_{0}$ $=5000$ atoms of rubidium at $k_{B} T=\mu$, where here $\mu$ $=6.25 \hbar \omega_{\text {ho }}$. In this case, one can see that the difference between the "strong" and "weak" transitions is not so impressive as in a bigger condensate since all damping strengths can be appreciated in the same scale.

We can conclude that at large $N_{0}$ we have actually two different phenomena. The strong transitions create temperature-induced resonances which can be observed in direct experiments. The background transitions give rise to Landau damping of the collective oscillations (see Sec. V B).

\section{A. Temperature-induced resonances}

Using the transition frequencies $\omega_{i k}$ and the corresponding damping strength $\gamma_{i k}$, we have calculated the response 


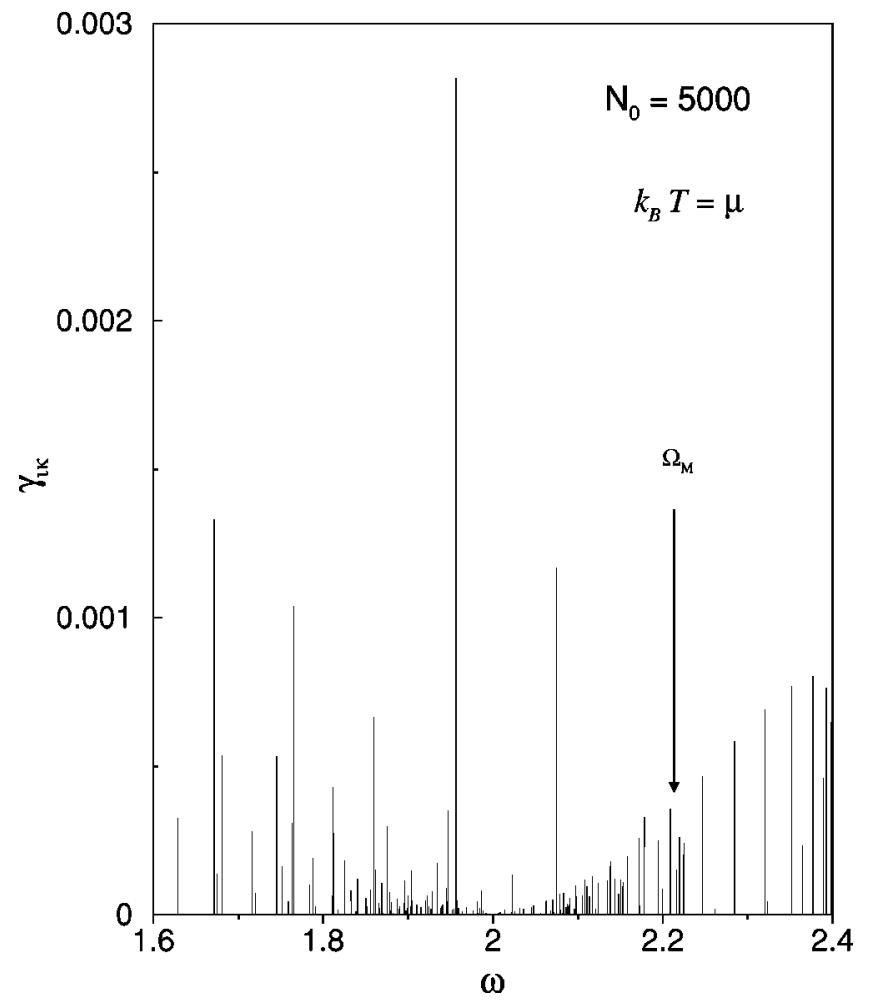

FIG. 2. Same as Fig. 1 but for $N_{0}=5000$ atoms at $k_{B} T=\mu$.

function $\alpha(\omega)$. At zero temperature, the response function $\alpha_{0}(\omega)$ given by Eq. (38) gives a resonance at the monopole frequency $\Omega_{M}=\sqrt{5} \omega_{\text {ho }}$ evaluated in the hydrodynamic regime. Due to interaction, thermal excited modes are coupled with the monopole. It means that when one excites the breathing mode of the condensate, the elementary excitations can give rise to other resonances at $\omega_{i k}$, which are the frequencies where $\operatorname{Re}\left[\alpha_{1}(\omega)\right]$ diverges [see Eq. (45)]. We will now discuss the conditions for the observation of these effects in actual experiments. In particular, we calculate the contribution of these resonances to the response function and estimate the associated strengths.

Let us study the resonances at $k_{B} T=\mu$ for $N_{0}=150000$ atoms of ${ }^{87} \mathrm{Rb}$. The behavior of the damping coefficients $\gamma_{i k}$ is analogous to that for 50000 condensate atoms (see Fig. 1), but in this case the difference between "strong" resonances and small background is even bigger: the dense background is not more resoluble in the scale of the strong resonances. There are five resonances that stand out from the others, and that we label as $\omega_{R}$ and $\gamma_{R}$ for the corresponding damping strength (see Table I for numerical values).

For perturbing frequencies close to the monopole $\omega$ $\sim \Omega_{M}$, the monopole susceptibility, Eq. (38), can be approximated to

$$
\alpha_{0}(\omega)=\frac{2 C}{m\left(\Omega_{M}-\omega\right)\left(\Omega_{M}+\omega\right)} \simeq A_{0} \frac{1}{\left(\Omega_{M}-\omega\right)},
$$

where $A_{0}=C /\left(m \Omega_{M}\right)$.

Analogously, $\alpha_{1}(\omega)$ near each resonance $\omega \sim \omega_{R}$ can be presented in the form $\alpha_{1}(\omega) \simeq A_{1} /\left(\omega_{R}-\omega\right)$. The ratio
TABLE I. Damping coefficients $\gamma_{R}$ (in units of $\omega_{\mathrm{ho}}$ ) of the "strong resonances" $\omega_{R}$ (in units of $\omega_{\mathrm{ho}}$ ) and relative intensities $\left|A_{1} / A_{0}\right|$ between the temperature-induced and the monopole resonance, for $N_{0}=150000$ condensate atoms of ${ }^{87} \mathrm{Rb}$ in a spherical trap with $a_{\mathrm{ho}}=0.791 \times 10^{-4} \mathrm{~cm}$ at $k_{B} T=\mu$.

\begin{tabular}{ccc}
\hline \hline$\omega_{R}$ & $\gamma_{R}$ & $\left|A_{1} / A_{0}\right|$ \\
\hline 1.9115 & 0.009268 & 0.063 \\
2.0252 & 0.004147 & 0.063 \\
2.1432 & 0.002102 & 0.127 \\
2.2576 & 0.001097 & 0.298 \\
2.3655 & 0.000545 & 0.020 \\
\hline
\end{tabular}

$A_{1} / A_{0}$ is a measure of the relative intensity between temperature-induced and monopole resonance.

Table I displays the numerical values of the relative intensity for each temperature-induced resonance $\omega_{R}$ with respect to the monopole one, for $N_{0}=150000$ atoms in the condensate at $k_{B} T=\mu$. The relative strength of the response function $\left(A_{1} / A_{0}\right)$ at $\omega_{R}$ depends not only on the damping coefficient $\gamma_{R}$ but also on $\left(\Omega_{M}^{2}-\omega_{R}^{2}\right)^{-1}$. It means that one mode $\omega_{R}$ will be easier to excite, i.e., the strength of the response will be bigger when it is close to the frequency of the monopole. Note also that the resonance strength increases with temperature through $\gamma_{R}$.

From Table I one can see that the biggest resonance occurs at $\omega_{R}=2.2576 \omega_{\text {ho }}$, which is resoluble from the monopole frequency $\Omega_{M}=2.234 \omega_{\text {ho }}$ and has a large enough relative strength to be observed. It means that by tuning the perturbation frequency $\omega$ to this value, a fluctuation of the fractional amplitude of oscillations can be observed.

In Fig. 3, we have plotted the frequency dependence of the real part of the response function $\alpha(\omega)$ calculated according to Eq. (48) for $N_{0}=150000$. The response function is given in arbitrary units, and frequency is in units of $\omega_{\text {ho }}$. The dashed line shows the monopole resonance at $\Omega_{M}$, whereas the other divergences of $\alpha(\omega)$ correspond to the temperature-induced resonances at $\omega_{R}$. From this figure one can see that the thermal induced resonances are quite distinct from each other and from the monopole one. Therefore,

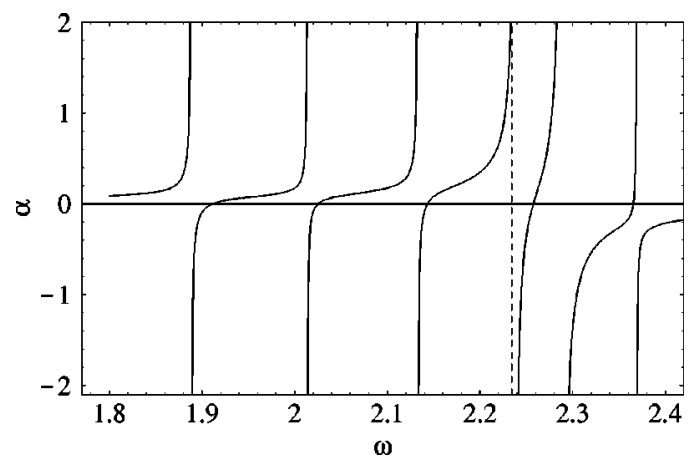

FIG. 3. Real part of the response function $\alpha(\omega)$ (in arbitrary units) as a function of $\omega$ (in units of $\omega_{\mathrm{ho}}$ ), for $N_{0}=150000$ at $k_{B} T=\mu$. The dashed line shows the monopole resonance at $\Omega_{M}$, whereas the other divergences of $\alpha(\omega)$ correspond to the temperature-induced resonances at $\omega_{R}$. 
temperature-induced resonances could be observed in experiments with good enough frequency resolution and good accuracy in the measurement of the radius fluctuations.

We would like to stress that the phenomenon we have discussed is related to quite delicate features of interaction between elementary excitations, and therefore its observation would give rich information about the properties of BoseEinstein condensed gases at finite temperature.

\section{B. Landau damping of collective modes}

From Fig. 1 one can see that the weak background transitions $\omega_{i k}$ have, generally speaking, very small frequency separation. To estimate this distance quantitatively, let us renominate the resonances by an index $i$ in the order of increasing value of $\omega$. Then, one can define the average distance between resonances $\overline{\Delta \omega}$ according to

$$
\overline{\Delta \omega}=\frac{\sum_{i} \gamma_{i}\left(\omega_{i+1}-\omega_{i}\right)}{\sum_{i} \gamma_{i}} .
$$

In a small interval around the collective oscillation $0.82 \Omega_{M}$ $<\omega_{i k}<1.18 \Omega_{M}$, we sum up all the transition frequencies allowed by the monopole selection rules and find the following values for the average distance between two consecutive transition frequencies: $\overline{\Delta \omega} / \omega_{\mathrm{ho}} \simeq 0.0006,0.001$, and 0.006 for $N_{0}=150000,50000$, and 5000, respectively. It is futile, of course, to try to resolve these resonances. Actually, there are reasons to believe that these resonances are smoothed and overlapped. First of all, a real trap cannot be exactly isotropic. This means that levels with different $m$ have slightly different energies; only levels with $m= \pm|m|$ are exactly degenerated. Therefore, each energy level with a given $l$ will be split on $l+1$ closer sublevels, making the energy spectrum more dense. Furthermore, all excitations at finite temperature have an associated finite lifetime. Excitations with $E \sim \mu$, which are the ones that mainly contribute in the "background" transitions, have the shortest lifetime. This can be accounted for phenomenologically by assuming that these levels have a finite Lorentzian width $\Delta$. That is, instead of $\delta$ functions in the equation for the damping rate (17), we will consider a Lorentzian distribution centered at $\omega_{i k}$ with a fixed width $\Delta: f_{L}\left(\omega_{i k}, \Delta\right)=\Delta /(2 \pi \hbar)\left[\left(\omega_{i k}\right.\right.$ $\left.\left.-\Omega_{\text {osc }}\right)^{2}+\Delta^{2} / 4\right]$. In this case the damping rate becomes a smooth function of $\Omega_{\text {osc }}$ and its value when $\Omega_{\text {osc }}=\Omega_{M}$ defines the Landau damping of the monopole oscillations. At conditions

$$
\overline{\Delta \omega} \ll \Delta \ll \omega_{\text {ho }},
$$

the damping rate will have only a weak dependence on the exact value of $\Delta$. In Fig. 4, we plot the dimensionless damping rate $\gamma / \Omega_{M}$ as a function of the Lorentzian width $\Delta$ (in units of $\omega_{\text {ho }}$ ) for $N_{0}=50000$ at different temperatures. The summation in Eq. (17) has been done over all resonances, excluding, of course, the "strong resonances" presented in Fig. 1. One can see that the $\Delta$ dependence is weak indeed in

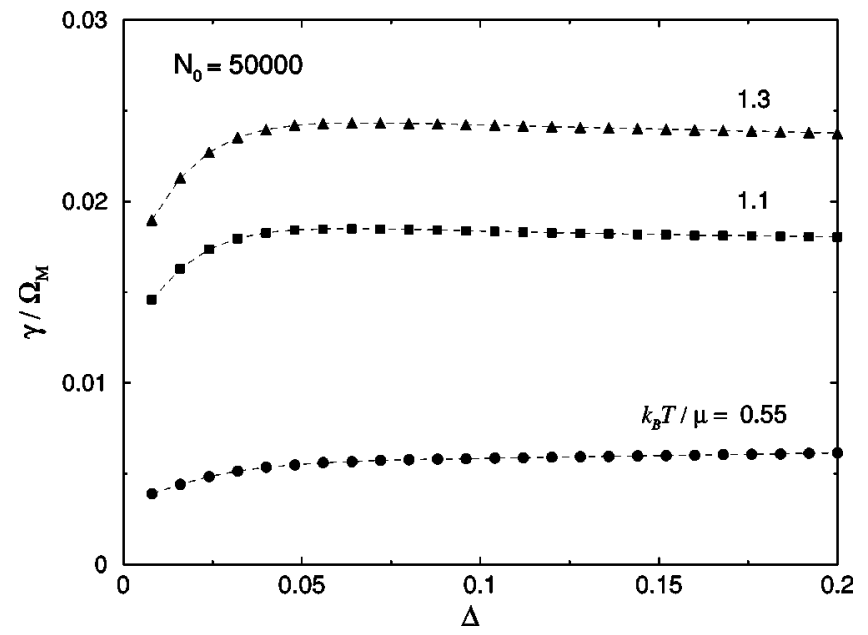

FIG. 4. Dimensionless damping rate $\gamma / \Omega_{M}$ as a function of the Lorentzian width $\Delta$ (in units of $\omega_{\text {ho }}$ ), for $N_{0}=50000{ }^{87} \mathrm{Rb}$ atoms in the spherical trap at different temperatures. Dashed lines are plotted as a guide for the eye. Solid dots, squares, and triangles correspond to the numerical calculation at $k_{B} T / \mu=0.55,1.1$, and 1.3 , respectively.

the interval $\Delta / \omega_{\mathrm{ho}}=0.05-0.2$ and $\gamma$ can be reliably extrapolated from this interval to the value $\Delta=0$. We take as Landau damping this extrapolated value of $\gamma$. One can estimate the accuracy of this extrapolation procedure to be of the order of $10 \%$ according to the change of $\gamma$ over this interval. In Fig. 5 , we plot the damping rate versus $k_{B} T / \mu$ for $N_{0}=150000$ and 50000 atoms in the condensate. As expected, Landau damping increases with temperature since the number of excitations available at thermal equilibrium is larger when $T$ increases. One can distinguish two different regimes in Fig. 5 , one at very low $T\left(k_{B} T \ll \mu\right)$ and the other at higher $T$. The behavior of the damping rate becomes linear at relatively small temperature $\left(k_{B} T \sim \mu\right)$ in comparison to the homogeneous system [4], where this regime occurs at $k_{B} T \gg \mu$. Moreover, the damping rate increases for a larger number of

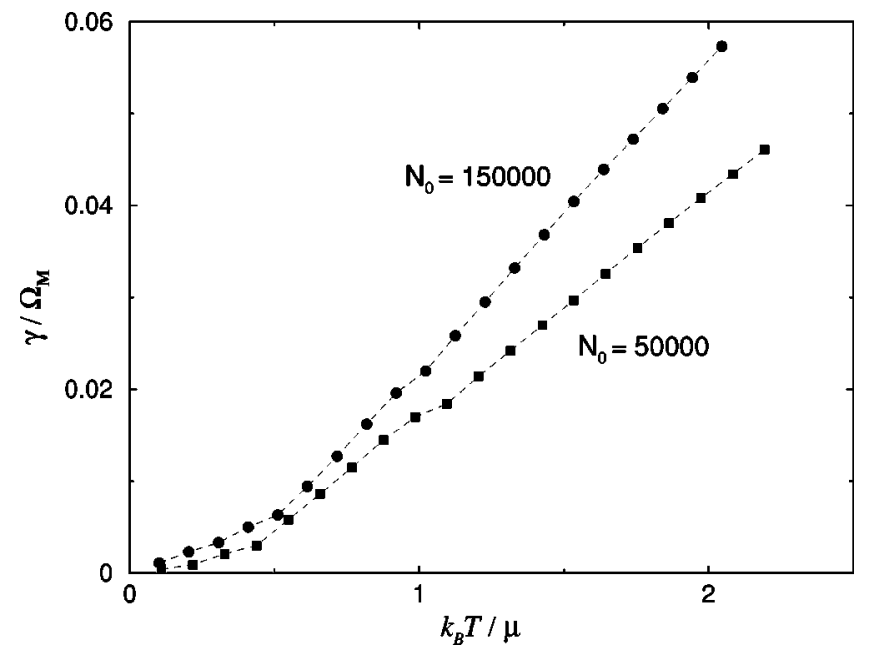

FIG. 5. Dimensionless damping rate $\gamma / \Omega_{M}$ as a function of $k_{B} T / \mu$ for $N_{0}=150000$ atoms (dots) and $N_{0}=50000$ atoms (squares). Dashed lines are plotted as a guide for the eye. 
condensed atoms because the density of states available to the system also increases. It is interesting to note that the order of magnitude of the damping rate is the same as the one previously estimated for a uniform gas [3-6] and for anisotropic traps $[7,8]$.

\section{SUMMARY}

We have considered the monopole oscillation of a Bosecondensed dilute atomic gas in an isotropic trap. First of all, we have calculated the normal modes of the condensate by solving the time-dependent Gross-Pitaevskii equation within Bogoliubov theory [18] and then we have used the formalism developed in Ref. [4] to calculate the matrix elements associated with the transitions between excited states allowed by the monopole selection rules. Within a first-order perturbation theory, we have studied the Landau damping of collective modes due to the coupling with thermal excited levels. We have developed the response function formalism to study the fluctuations of the system due to an external perturbation. The contribution of the elementary excitations has been in- troduced also perturbatively as in the calculation of the damping strength, and we have derived analytic equations for the response function at zero temperature and at the lowtemperature regime. We have seen that when the condensate oscillates with the monopole mode and a small perturbation to the trap frequency is applied, one can excite new resonances at the transition frequencies. These thermal-induced resonances are coupled with the monopole due to interaction effects. One cannot exclude a priori the possibility to observe such resonances also in anisotropic traps. This problem deserves further investigation. Observation of these resonances would give important and unique information about the interaction between elementary excitations in BoseEinstein condensed gases.

\section{ACKNOWLEDGMENTS}

We thank F. Dalfovo, P. Fedichev, and S. Stringari for helpful discussions. M.G. thanks the Istituto Nazionale per la Fisica della Materia (Italy) for financial support.
[1] D.S. Jin, M.R. Matthews, J.R. Ensher, C.E. Wieman, and E.A. Cornell, Phys. Rev. Lett. 78, 764 (1997).

[2] M.-O. Mewes, M.R. Andrews, N.J. van Druten, D.M. Kurn, D.S. Durfee, C.G. Townsend, and W. Ketterle, Phys. Rev. Lett. 77, 988 (1996).

[3] W.V. Liu and W.C. Schieve, e-print cond-mat/9702122.

[4] L.P. Pitaevskii and S. Stringari, Phys. Lett. A 235, 398 (1997).

[5] W.V. Liu, Phys. Rev. Lett. 79, 4056 (1997).

[6] S. Giorgini, Phys. Rev. A 57, 2949 (1998).

[7] P.O. Fedichev, G.V. Shlyapnikov, and J.T.M. Walraven, Phys. Rev. Lett. 80, 2269 (1998).

[8] P.O. Fedichev and G.V. Shlyapnikov, Phys. Rev. A 58, 3146 (1998).

[9] N.N. Bogoliubov, J. Phys. (USSR) 11, 23 (1947).

[10] E.P. Gross, Nuovo Cimento 20, 454 (1961); E.P. Gross, J. Math. Phys. 4, 195 (1963).

[11] L.P. Pitaevskii, Zh. Éksp. Teor. Fiz. 40, 646 (1961) [Sov. Phys. JETP 13, 451 (1961)].

[12] W. Ketterle, D.S. Durfee, and D.M. Stamper-Kurn, e-print cond-mat/9904034.

[13] M. Edwards, P.A. Ruprecht, K. Burnett, R.J. Dodd, and C.W. Clark, Phys. Rev. Lett. 77, 1671 (1996); P.A. Ruprecht, M. Edwards, K. Burnett, and C.W. Clark, Phys. Rev. A 54, 4178 (1996); M. Edwards, R.J. Dodd, C.W. Clark, and K. Burnett, J. Res. Natl. Inst. Stand. Technol. 101, 553 (1996).

[14] K.G. Singh and D.S. Rokhsar, Phys. Rev. Lett. 77, 1667 (1996).

[15] B.D. Esry, Phys. Rev. A 55, 1147 (1997).

[16] D.A.W. Hutchinson, E. Zaremba, and A. Griffin, Phys. Rev. Lett. 78, 1842 (1997).

[17] L. You, W. Hoston, and M. Lewenstein, Phys. Rev. A 55, R1581 (1997).

[18] F. Dalfovo, S. Giorgini, M. Guilleumas, L.P. Pitaevskii, and S. Stringari, Phys. Rev. A 56, 3840 (1997).
[19] S. Stringari, Phys. Rev. Lett. 77, 2360 (1996).

[20] When $N a / a_{\text {ho }}$ is large, one can use the hydrodynamic approximation for the functions $u$ and $\mathrm{v}$ of the low-energy excitations [see L.P. Pitaevskii, Recent Progress in Many-Body Theories, edited by D. Nielson and R. Bishop (World Scientific, Singapore, 1998), p. 3]. However, we have found that the matrix elements $A_{i k}$, defined in Sec. III, are quite sensitive to the accuracy of these functions. Hydrodynamics can give values for $\gamma_{i k}$ an order of magnitude smaller than the values calculated numerically by using the Bogoliubov functions (4) and (5). On the contrary, it is completely safe to use hydrodynamics to calculate the response function at zero temperature $\alpha_{0}(\omega)$ (see Sec. IV), since the difference between hydrodynamics and exact values of the monopole frequency $\Omega_{M}$ is very small.

[21] Beliaev decay of an elementary excitation into a pair of excitations (S.T. Beliaev, Zh. Éksp. Teor. Fiz. 7, 469 1958) [Sov. Phys. JETP 34, 322 (1958)] is not active for the lowest-energy modes in the case of the trapping potential because of the discretization of levels.

[22] L.D. Landau and E.M. Lifshitz, Statistical Physics, Course of Theoretical Physics (Pergamon Press, Oxford, 1970), Vol. 5.

[23] L.P. Pitaevskii, Phys. Lett. A 229, 406 (1997).

[24] F. Dalfovo, C. Minniti, and L.P. Pitaevskii, Phys. Rev. A 56, 4855 (1997).

[25] F. Dalfovo, S. Giorgini, L.P. Pitaevskii, and S. Stringari, Rev. Mod. Phys. 71, 463 (1999).

[26] A.A. Abrikosov, L.P. Gorkov, and I. Ye. Dzyaloshinskii, Quantum Field Theoretical Methods in Statistical Physics (Pergamon Press, Oxford, 1965).

[27] S. Giorgini, L.P. Pitaevskii, and S. Stringari, J. Low Temp. Phys. 109, 309 (1997).

[28] In this paper we do not include the so-called Popov's selfconsistent correction in Eq. (1). See, for example, Ref. [27]. 
[29] We have neglected all the transitions that involve the lowest dipole mode $(l=1, n=0)$ because in an external harmonic potential this mode, corresponding to the oscillation of the center of mass, is unaffected by the interatomic forces and then the transition probability due to interaction effects (11) must be zero $[19,30]$. However, the perturbation formalism we have presented in Sec. III does not take into account this physical consideration automatically and, therefore, we have omitted by hand all transitions with the dipole mode.

[30] H. Stoof, J. Low Temp. Phys. 114, 11 (1999). 